\title{
Análise de componentes principais de pães de forma formulados com farinha de yacon (Smallanthus sonchifolius (Poepp.) H. Rob.)
}

\author{
Priscilla Moura Rolim ${ }^{1}$, Silvana Magalhães Salgado², Vivianne Montarroyos Padilha ${ }^{1}$, \\ Alda Verônica Souza Livera ${ }^{2}$, Nonete Barbosa Guerra², Samara Alvachian Cardoso Andrade ${ }^{3}$
}

\section{RESUMO}

O yacon, tubérculo de origem andina, vem sendo cada vez mais aplicado na tecnologia de alimentos, em virtude de suas propriedades funcionais, por ser fonte de inulina e fruto-oligossacarídeos, considerados prebióticos. Foram preparadas três formulações de pães, sendo uma padrão e duas testes contendo farinha de yacon, denominadas de amostra P (pão padrão, sem farinha de yacon), amostra A (pão com 6\% de farinha de yacon e 3\% de gordura) e amostra B (pão com 11\% de farinha de yacon sem adição de gordura). Para a avaliação sensorial foi realizada a Análise Descritiva Quantitativa (ADQ), utilizando-se 11 provadores treinados, os quais definiram 10 atributos sensoriais (aroma, cor, porosidade e uniformidade do miolo, sabor, textura, umectância, untuosidade, adesividade e qualidade global). Também foi realizada análise física dos pães, por meio da caracterização cromática. Os resultados da ADQ foram analisados por análise de variância e testes de médias de Duncan, a 5\% de significância. Os resultados da ADQ foram representados graficamente por análise multivariada de análise de componentes principais (ACP). Não houve diferença entre as amostras A e B em relação aos atributos porosidade, textura, sabor e qualidade global. A amostra A obteve média maior em atributos sensoriais indesejáveis como aderência, enquanto a amostra B apresentou maior média para o atributo umectância, devido ao maior teor de yacon. Na ACP, a soma de componentes principais 1 e 2 foi de $97,59 \%$, ou seja, a variabilidade entre as amostras foi quase totalmente explicada por estes dois componentes. Os resultados obtidos demonstram o grande potencial para o desenvolvimento de novos produtos utilizando alimentos fontes de prebióticos, como os frutanos do yacon.

Palavras-chave: Análise sensorial, prebióticos, análise de componente principal, yacon.

\section{ABSTRACT}

\section{Principal component analysis of sliced bread formulated with yacon meal (Smallanthus sonchifolius(Poepp.) H. Rob.))}

The yacon, tuber vegetable crop of Andean origin, is increasingly being used in food technology because of its functional properties, as a source of inulin and fructooligosaccharides, which are considered prebiotics. Samples of bread made out of yacon were analyzed. Sample P (Standard bread, without yacon flour), sample A (6\% yacon flour and 3\% fat) and sample B (11\% yacon flour, without fat). Quantitative Descriptive Analysis (QDA) was carried out for sensory evaluation; 11 experienced tasters participated and defined 10 sensory attributes (smell, color, crumb porosity and uniformity, flavor, texture, humectancy, unctuousness, adhesiveness and overall quality). Bread physical analysis was also carried out by chromatic characterization. QDA results were analyzed by analysis of variance and Duncan's test of means, at 5\% significance level. QDA results were graphically represented by multivariate principal component

\footnotetext{
Recebido para publicação em março de 2009 e aprovado em novembro de 2009

${ }^{1}$ Nutricionistas, Mestres. Programa de Pós-graduação em Nutrição, Centro de Ciências da Saúde, Universidade Federal de Pernambuco (UFPE), Cidade Universitária, 50670901, Recife, Pernambuco, Brasil. priscillanutri@hotmail.com (Autor para correspondência), vivianne_padilha@yahoo.com.br

${ }^{2}$ Nutricionistas, Doutoras. Programa de Pós-graduação em Nutrição, Centro de Ciências da Saúde, UFPE, Cidade Universitária, 50670-901, Recife, Pernambuco, Brasil. silvanasalgado@ufpe.br, aldalivera@ufpe.br,nonete@globo.com

${ }^{3}$ Engenheira Química, Doutora. Programa de Pós-graduação em Nutrição, Centro de Ciências da Saúde, UFPE, Cidade Universitária, 50670-901, Recife, Pernambuco, Brasil. samaraandrade@uol.com.br
} 
analysis (PCA). No difference was found between samples A and B for porosity, texture, flavor, and overall quality. Sample A showed the highest means for undesirable sensory attributes such as adherence, whereas sample B showed the highest mean for humectancy due to the highest content of yacon, which is a highly hygroscopic product. In PCA, the sum of the main components 1 and 2 was $97.59 \%$, that is, the variability between the samples is almost totally explained by these two components. The results demonstrate the great potential for the development of new products using prebiotic food source such as the fructans from yacon.

Key words: Sensory analysis, prebiotics, principal component analysis, yacon.

\section{INTRODUÇÃO}

Prebióticos como os frutanos, inulina e fruto-oligossacarídeos (FOS) estão sendo utilizados, na sua forma isolada ou por meio de alimentos fonte, como ingredientes alimentícios, por razões nutricionais e tecnológicas.

Como exemplo tem-se o yacon (Smallanthus sonchifolius), fonte promissora de frutanos (Quinteros, 2000), que a partir de suas raízes tuberosas é possível obter farinha, aplicável a produtos com baixo teor de gordura e açúcar, reduzindo o valor calórico, além de propiciar benefícios fisiológicos.

Os efeitos que desempenham no organismo estão relacionados ao processo fermentativo no cólon, como o crescimento de bactérias não patógenas produtoras de ácidos graxos de cadeia curta que podem interferir no metabolismo lipídico (Meier \& Lochs, 2007). Outros benefícios são o de apresentar baixa cariogenicidade, aumentar a absorção de cálcio, magnésio e ferro, estimular a produção de vitaminas do complexo B, e inibir o estágio inicial do câncer de cólon, regularizando a função intestinal (Roberfroid, 2005).

Do ponto de vista tecnológico, os frutanos são utilizados como substituto da gordura e do açúcar, tendo em vista a capacidade da inulina de formar géis estáveis e a função umectante e edulcorante dos FOS, semelhante à sacarose, porém com aporte calórico reduzido (1,5kcal/g) (Meier \& Lochs, 2007).

Diante destas constatações, cresce o interesse pelo desenvolvimento de "novos produtos com propriedades de alegações funcionais”, que atendam aos anseios dos consumidores, ultimamente bastante exigentes quanto aos padrões de qualidade dos alimentos e conscientes da relação existente entre alimentação e saúde.

Um exemplo do crescimento de novos produtos, no Brasil, é o segmento de panificação. O pão, considerado um alimento popular de elevado consumo na forma de lanches ou acompanhando as refeições, é bastante apreciado, devido às suas características organolépticas e disponibilidade (Battochio, 2006). Atualmente existe grande variedade de pães enriquecidos com fibras alimentares de diversas fontes, propiciando à tecnologia de alimen- tos a criação de novas formulações, com vista à obtenção de produtos diferenciados no mercado.

Considerando os possíveis efeitos fisiológicos dos frutanos e a importância do pão na alimentação do brasileiro, este trabalho teve como objetivo avaliar a qualidade sensorial de pães formulados com farinha de yacon.

\section{MATERIAL E MÉTODOS}

Os ingredientes utilizados na fabricação dos pães foram adquiridos no comércio local de Recife, PE, observando-se a adequação de apresentação e o prazo de validade. As raízes de yacon foram obtidas no Ceasa (Centro de Abastecimento da Região Metropolitana do Recife, PE).

Os ingredientes utilizados para fabricação das amostras de pães estão demonstrados na Tabela 1, correspondendo à amostra $\mathrm{P}$ (pão padrão sem farinha de yacon), amostra A (pão adicionado de 6\% de farinha de yacon) e amostra B (pão adicionado de 11\% de farinha de yacon).

\section{Processamento da farinha de yacon}

Inicialmente o yacon in natura foi lavado em água corrente e sanitizado em solução clorada a $200 \mathrm{mg} \mathrm{L}^{-1}$ por 10 minutos. A seguir, o yacon foi fatiado manualmente em lâminas finas, de aproximadamente $1 \mathrm{~cm}$, e imerso em solução de cloreto de cálcio a $1 \%$ por 30 minutos. O mesmo foi submetido à secagem em estufa ventilada com circulação de ar, marca Tecnal, a $55^{\circ} \mathrm{C}$ por 24 horas e, posteriormente, triturado em multiprocessador marca Arno para a obtenção da farinha com granulometria de 20 mesh. A farinha foi embalada em sacos transparentes estéreis e armazenada em dessecador a $15^{\circ} \mathrm{C}$.

\section{Análise Descritiva Quantitativa}

\section{Seleção dos Provadores}

Onze provadores foram escolhidos para compor a equipe descritiva. Para avaliar o poder discriminativo de cada voluntário, foi utilizado o teste de sensibilidade aos gostos básicos (Teste Threshold de Reconhecimento) e foram selecionados os provadores que acertaram no mínimo 50\% dos gostos (Anzaldua-Morales, 1994). 
Tabela 1. Proporção dos ingredientes nas formulações dos pães de três amostras

\begin{tabular}{lccc}
\hline & \multicolumn{3}{c}{$\begin{array}{c}\text { Formulações dos Pães (\%) } \\
\text { Amostras* }\end{array}$} \\
\cline { 2 - 4 } Ingredientes & $\mathbf{P}$ & $\mathbf{A}$ & $\mathbf{B}$ \\
\hline Farinha de trigo & 100 & 100 & 100 \\
Farinha de yacon & 0 & 6 & 11 \\
Sal & 2 & 2 & 2 \\
Açúcar cristal & 1 & 1 & 1 \\
Fermento biológico & 4 & 4 & 4 \\
Leite integral UHT & 60 & 62 & 63 \\
Gordura vegetal hidrogenada & 6 & 3 & 0 \\
\hline
\end{tabular}

${ }^{*} \mathrm{P}=$ Padrão, $\mathrm{A}=$ Amostra com $6 \%$ de yacon e $3 \%$ de gordura, $\mathrm{B}=$ Amostra com $11 \%$ de yacon e sem gordura.

\section{Definição da Terminologia Descritiva}

As amostras foram apresentadas aos provadores, eles descreveram as similaridades e diferenças entre cada amostra com relação à aparência global, ao aroma, à cor, ao sabor e à textura. Em seguida, de posse de ampla lista de termos descritivos levantados na literatura, sob a supervisão do líder da equipe, os provadores discutiram o significado de cada termo. Dessa forma, pelo consenso da equipe sensorial foi elaborada uma ficha de avaliação sensorial contemplando a definição de cada termo descritivo (Tabela 2).

A ficha de avaliação apresenta uma escala não estruturada para cada termo descritivo levantado, a qual foi composta de uma linha de $9 \mathrm{~cm}$, tendo expressões quantitativas (pontos-âncora) nas extremidades esquerda (equivalente ao ponto um) e direita (equivalente ao ponto nove) com os termos: "claro”, “escuro”, “pouco”, “muito”, "leve”, "pesado”, entre outros. Os provadores, por meio de um traço vertical na escala, escolhiam a melhor posição que refletisse a sua avaliação para cada termo descritivo. Os valores foram obtidos medindo-se a distância entre os pontos-âncoras da extremidade esquerda e o traço vertical feito pelo provador, com auxílio de uma régua (Brasil, 2005).

\section{Avaliação das Amostras}

Os pães foram servidos em recipiente branco, identificado com números de três algarismos, em blocos completos balanceados, com três repetições, acompanhados de água à temperatura ambiente, juntamente com a ficha gerada com os termos descritores, em cabines individuais, no Laboratório de Análise Sensorial do Departamento de Nutrição da Universidade Federal de Pernambuco. Para avaliação do aroma foi oferecido ao provador um recipiente fechado, contendo pedaços do pão em estudo. No critério untuosidade, o provador dispôs de manteiga à temperatura ambiente para ser passada sobre o pão.

Os dados obtidos foram submetidos à análise de variância (ANOVA) e os tratamentos, comparados utilizando-se do teste de comparação de médias de Duncan, a 5\% de significância. Para tal, foi utilizado o software Statistica for Windows (Statsoft, 2001). As respostas sensoriais foram avaliadas pela metodologia da Análise de Componentes Principais (ACP), utilizando-se de procedimentos sugeridos por Minim (2006).

\section{Caracterização Cromática dos Pães}

A avaliação das cores dos pães foi realizada por meio do sistema de leitura de três parâmetros, o CIELAB, também conhecido como espaço L*a* b* (Mcguire, 1992). O parâmetro $\mathrm{L}^{*}$ está associado à luminosidade das amostras e pode variar de 0 a 100, e os valores mais altos de L* (próximos de 100) caracterizam as amostras mais claras e os menores valores de L* (menores que 50 ) as mais escuras. A coordenada cromática $\mathrm{a}^{*}$ está associada à dimensão verde-vermelho; valores positivos de a* indicam amostras na região do vermelho; e valores negativos de a*

Tabela 2. Definições dos termos descritivos para as amostras de pães

\begin{tabular}{ll}
\hline Descritores & Definições \\
\hline Aroma característico & Aroma característico de pão de forma quente \\
Cor do miolo & Cor característica amarelo-clara de pão de fôrma \\
Porosidade & Tamanho das cavidades formadas; deformações no miolo; presença de buracos \\
Uniformidade do miolo & Homogeneidade da massa em relação à sua aparência global \\
Textura & Número de mastigações necessárias antes da deglutição \\
Sabor característico & Sabor característico de pão de forma \\
Umectância & Sensação provocada pela quantidade de água no alimento \\
Aderência & Força necessária para superar a tração entre o alimento e o palato e/ou dentes \\
Untuosidade & Facilidade com que a manteiga desliza na superfície da amostra com auxílio de espátula \\
\hline
\end{tabular}


amostras na região do verde. A coordenada cromática b* está associada à dimensão azul-amarelo, valores positivos de b* indicam amostras na região do amarelo e valores negativos de b* indicam amostras na região do azul.

\section{RESULTADOS E DISCUSSÃO}

\section{Análise Descritiva Quantitativa (ADQ)}

Os resultados descritos na Tabela 3 demonstraram que as amostras experimentais obtiveram médias significativamente superiores $(\mathrm{p}<0,05)$ à da amostra padrão nos atributos cor do miolo, textura, umectância e aderência. Para os atributos porosidade, textura, sabor característico e qualidade global não houve diferença significativa entre as amostras A e B.

Embora a amostra B (11\% de farinha de yacon) tenha sido elaborada com maior teor de farinha de yacon, foi a amostra A (6\% de farinha de yacon) que obteve a maior média no atributo sensorial aderência, considerado pouco desejado pelo consumidor. A maior aderência apresentada pela amostra A foi possivelmente devida à junção da farinha de yacon com a gordura utilizada no pão A. A farinha de yacon é bastante higroscópica e sua própria umidade, combinada com a gordura, pode ter contribuído para a aderência acentuada da amostra A.

Com relação ao atributo umectância, a amostra B obteve a maior média, provavelmente em função de o maior teor de frutanos resultar numa elevada capacidade de retenção de água pelo produto. A interação frutano-água também influenciou o atributo untuosidade, pois os pães com farinha de yacon apresentaram menor capacidade de deslizamento da manteiga sobre sua superfície.

A distribuição gráfica dos resultados apresentados na Figura 1 confirma que as amostras experimentais apresentaram maior semelhança entre si do que o pão padrão. Apesar das médias para qualidade global terem sido um pouco mais elevadas na análise do pão padrão, a aceitação dos demais pães contendo farinha de yacon pode ser considerada boa, uma vez que gerou médias superiores a 6,0.

No gráfico da ACP (Figura 2) cada amostra de pão é representada por um ponto, no qual cada ponto corresponde ao valor médio atribuído pela equipe sensorial, em cada repetição. Amostras similares ocupam regiões próximas no gráfico e são caracterizadas pelos vetores (atributos) que se apresentam mais próximos a elas. Podese observar que as amostras ficaram bem distintas umas das outras, marcadas pelas localizações bem definidas de cada uma nos gráficos.

Ao analisar o primeiro componente principal que reproduz $87,65 \%$ da variabilidade entre as amostras, constata-se que, conforme esperado, o pão padrão obteve mais escores positivos, sendo melhor representado pelos atributos sensoriais: sabor característico, uniformidade do miolo, porosidade, aroma característico, untuosidade e qualidade global, confirmado pelas maiores e significativas notas obtidas em todos estes atributos (Tabela 3).

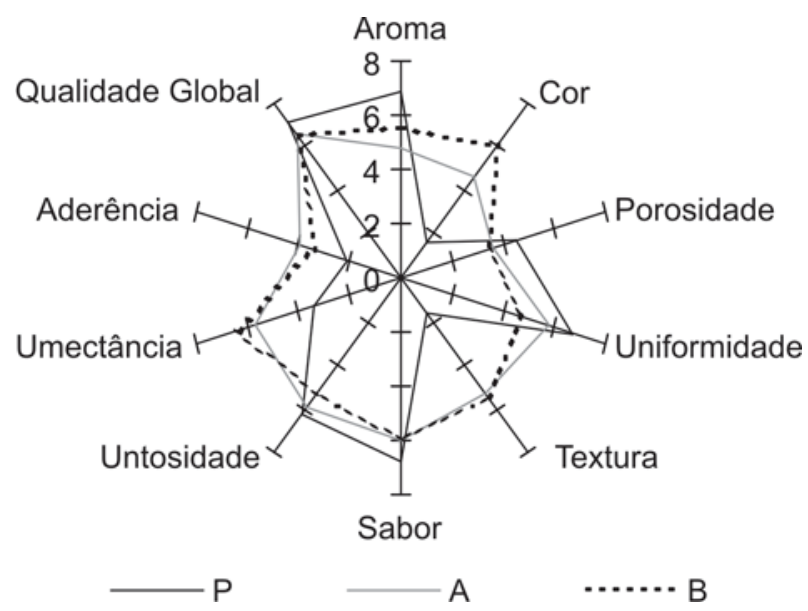

Figura 1. Perfil sensorial para as amostras de pão. $\mathrm{P}=$ Padrão, $\mathrm{A}$ $=$ Amostra com $6 \%$ de yacon e $3 \%$ de gordura, $\mathrm{B}=$ Amostra com $11 \%$ de yacon e sem gordura.

Tabela 3. Médias de intensidade atribuída aos atributos sensoriais das amostras dos pães pela equipe de provadores

\begin{tabular}{lccc}
\hline \multirow{2}{*}{ Atributos Sensoriais } & & Amostras ${ }^{*}$ & B \\
\cline { 2 - 4 } & $\mathbf{P}$ & $\mathbf{A}$ & $5,53 \mathrm{~b}$ \\
Aroma característico & $6,86 \mathrm{a}$ & $4,82 \mathrm{c}$ & $6,01 \mathrm{a}$ \\
Cor do miolo & $1,55 \mathrm{c}$ & $4,60 \mathrm{~b}$ & $3,35 \mathrm{~b}$ \\
Porosidade & $4,49 \mathrm{a}$ & $3,60 \mathrm{~b}$ & $4,67 \mathrm{c}$ \\
Uniformidade do miolo & $6,73 \mathrm{a}$ & $5,75 \mathrm{~b}$ & $5,51 \mathrm{a}$ \\
Textura & $1,67 \mathrm{~b}$ & $5,34 \mathrm{a}$ & $5,91 \mathrm{~b}$ \\
Sabor característico & $6,82 \mathrm{a}$ & $5,96 \mathrm{~b}$ & $6,33 \mathrm{a}$ \\
Umectância & $3,43 \mathrm{c}$ & $5,68 \mathrm{~b}$ & $3,38 \mathrm{~b}$ \\
Aderência & $2,12 \mathrm{c}$ & $3,92 \mathrm{a}$ & $5,32 \mathrm{c}$ \\
Untuosidade & $6,28 \mathrm{a}$ & $5,94 \mathrm{~b}$ & $6,51 \mathrm{~b}$ \\
Qualidade global & $7,10 \mathrm{a}$ & $6,54 \mathrm{~b}$ &
\end{tabular}

${ }^{*} \mathrm{P}=$ Padrão, $\mathrm{A}=$ Amostra com 6\% de yacon e 3\% de gordura, $\mathrm{B}=$ Amostra com $11 \%$ de yacon e sem gordura.

Médias seguidas de letras iguais na horizontal não diferem significativamente entre si a 5\% de significância pelo teste de Duncan. 
Quanto ao pão B (11\% de farinha de yacon), apesar de apresentar mais escores negativos do que positivos, obteve boa qualidade global, tendo sido caracterizado pelos atributos cor do miolo, textura e umectância. Além disso, apresentou cor amarela mais escura, textura mais pesada e maior umectância, também confirmado pelas notas da Tabela 3.

No segundo componente principal, que explica 8,91\% das informações, pode-se observar que o pão A (6\% de farinha de yacon) possui escores positivos, caracterizando esse produto pelos atributos aderência, uniformidade e untuosidade.

A maior parte das variações que ocorreram entre as amostras foi explicada pelo Componente Principal 1, mas quando avaliada associada ao Componente Principal 2 observou-se que os dois componentes explicaram 97,59\% das informações contidas nos valores médios das 10 variáveis sensoriais; ou seja, a variabilidade entre as amostras pôde ser bem explicada utilizando apenas esses dois eixos (Figura 2).

\section{Caracterização cromática das amostras}

Na Tabela 4 verificam-se os valores do parâmetro L*e das coordenadas cromáticas a* e b* da análise de cor das amostras dos pães. Essas amostras se apresentaram nas regiões do verde e do amarelo e com valores do parâmetro $\mathrm{L}^{*}$ acima de 50 ( $\mathrm{L}^{*}>50$ ), caracterizando-as como amostras claras. Observa-se que a amostra B (11\% de farinha de yacon) teve valor de $\mathrm{L}^{*}$ significativamente menor que as amostras $\mathrm{P}$ e $\mathrm{A}$, indicando que essa amostra apresentou-se mais escura, resultado da coloração do yacon após reações de escurecimento enzimático.

O pão com $6 \%$ de farinha de yacon na coordenada cromática $\mathrm{a}^{*}$ teve valor inferior aos pães padrão e $\mathrm{B}$, ou seja, sua intensidade para o verde foi mais acentuada, no entanto, o pão padrão apresentou cor verde significativamente $(\mathrm{p}<0,05)$ mais intensa.

Na coordenada $b^{*}$ pode-se constatar que as três amostras não diferiram significativamente entre si $(\mathrm{p}<0,05)$, apresentando coloração mais amarelada, uma vez que seus valores foram positivos.

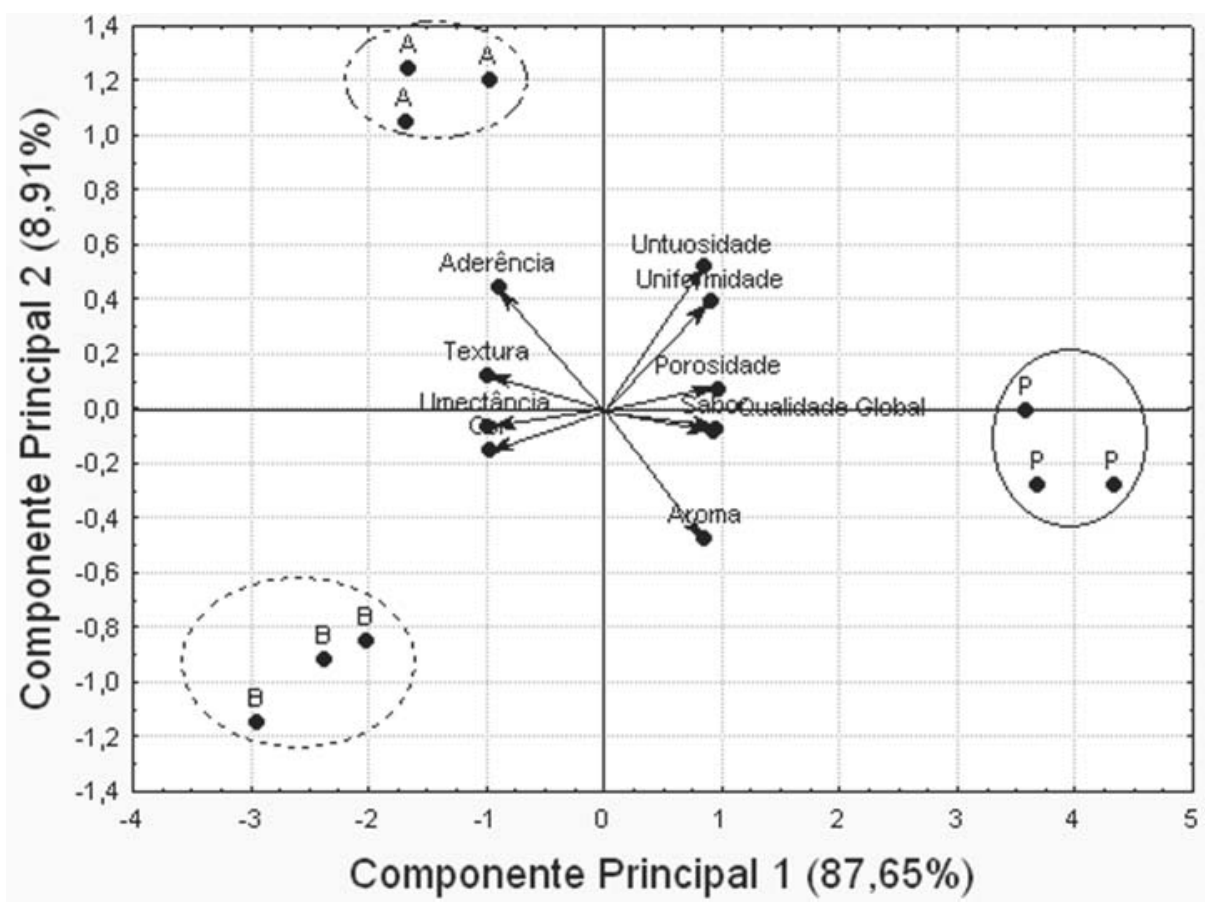

Figura 2. Bidimensional da Análise de Componentes Principais dos termos descritores das amostras de pão. $\mathrm{P}=\mathrm{Padrão}, \mathrm{A}=\mathrm{Amostra}$ com $6 \%$ de yacon e $3 \%$ de gordura, $\mathrm{B}=$ Amostra com $11 \%$ de yacon e sem gordura.

Tabela 4. Valores obtidos do parâmetro L* e das coordenadas cromáticas a* e b* da análise de cor das amostras dos pães

\begin{tabular}{lccc}
\hline Amostras $^{1}$ & $\mathbf{L}^{*}$ & $\mathbf{a}^{*}$ & $\mathbf{b}^{*}$ \\
\hline $\mathrm{P}$ & $71,48 \mathrm{a}$ & $-1,92 \mathrm{a}$ & $+18,95 \mathrm{a}$ \\
$\mathrm{A}$ & $71,06 \mathrm{a}$ & $-1,56 \mathrm{~b}$ & $+20,95 \mathrm{a}$ \\
$\mathrm{B}$ & $68,60 \mathrm{~b}$ & $-1,77 \mathrm{~b}$ & $+22,32 \mathrm{a}$ \\
\hline
\end{tabular}

${ }^{1} \mathrm{P}=$ Padrão, $\mathrm{A}=$ Amostra com $6 \%$ de yacon e $3 \%$ de gordura, $\mathrm{B}=$ Amostra com $11 \%$ de yacon e sem gordura.

Médias seguidas de letras iguais na vertical não diferem significativamente entre si a 5\% de significância pelo teste de Duncan.

Rev. Ceres, Viçosa, v. 57, n.1, p. 012-017, jan/fev, 2010 
Comparando os resultados de colorimetria com o atributo sensorial cor do miolo, observou-se que todas as amostras diferiram significativamente entre si. A cor do miolo do pão padrão apresentou-se mais clara, comprovada pelo valor $\mathrm{L}^{*}$ demonstrado na Tabela 4 . No entanto, para as amostras A e B verificou-se escurecimento do miolo proporcional à adição de farinha de yacon.

A presença de compostos fenólicos, como o ácido clorogênico, e do aminoácido L-triptofano torna os tubérculos do yacon suscetíveis à reação de escurecimento causada pelas enzimas peroxidase (POD) e polifenoloxidase (PPO). Nessa reação ocorre a formação de melanina (pigmento escuro), que deprecia a qualidade do produto (Neves, 2007). O controle dessa reação pode ser feito por meio da desidratação, do armazenamento a baixas temperaturas, do tratamento térmico, da eliminação do oxigênio do meio, da utilização de agentes químicos, dentre outros (Padilha et al., 2009).

\section{CONCLUSÕES}

É possível concluir que nas porcentagens de farinha de yacon utilizadas (6 e 11\%) não há diferença entre os pães padrão e testes quanto aos atributos porosidade, textura, sabor e qualidade global. Entretanto, o pão com $6 \%$ de farinha de yacon apresenta a maior média para o atributo aderência, fato indesejável para a qualidade sensorial de pães. A Análise Descritiva Quantitativa evidencia que as características sensoriais das amostras são dependentes da variação dos ingredientes utilizados nas formulações. Portanto, a fabricação de pães com propriedades prebióticas contendo farinha de yacon é promissora, uma vez que os atributos sensoriais avaliados são considerados satisfatórios.

\section{REFERÊNCIAS}

Anzaldua-Morales A (1994) La evalucación sensorial de los alimentos en la teoria y la práctica. Zaragoza, Editorial Acribia. p.21-23.

Battochio JR (2006) Perfil sensorial de pão de forma integral. Ciência e Tecnologia de Alimentos, 26:428-433.

Brasil (2005) Ministério da Saúde. Agência Nacional de Vigilância Sanitária. Métodos Físico-químicos para análise de alimentos/ Ministério da Saúde. Brasília, Agência Nacional de Vigilância Sanitária -Ministério da Saúde, p. 311-313.

Minim VPR (2006) Análise sensorial: estudos com consumidores. Viçosa, Ed. UFV. 225p.

Mcguire RG (1992) Reporting of objective color measurements. Horticultural Science, 27:1254-1555.

Meier R \& Lochs H (2007) Pre-and probiotics. Ther umsch (therapeutishe umshau. Revue Therapeutique, 64:161-169.

Neves VA (2007) Polyphenoloxidase from yacon roots (Smallanthus sonchifolius). Journal of Agricultural and Food Chemistry, 55:2424-30.
Padilha VM, Rolim PM, Salgado SM, Livera AVS \& Oliveira MG (2009) Avaliação do tempo de secagem e da atividade de óxidoredutases de yacon (Smallanthus sonchifolius) sob tratamento químico. Ciência Rural, 39:2178-2184.

Roberfroid MB (2005) Introducing inulin-type fructans. British Journal of Nutrition, 93:13-25.

Quinteros T (2000) Produção com tratamento enzimático e avaliação do suco de yacon. Tese de Doutorado. Universidade Estadual de Campinas, Campinas. 120p.

Statsoft (2001) Statistica for Window - Computer programa manual. Versão 6.0 Tulsa, Statsoft Inc. 